\title{
Effect of Zn:Se Ratio on the Properties of Sprayed ZnSe Thin Films
}

\author{
M. Öztaş, M. BediR*, Ö.F. BakkaloĞlu and R. Ormanci \\ University of Gaziantep, Department of Engineering Physics \\ 27310-Gaziantep, Turkey
}

(Received June 24, 2004; revised version December 2, 2004)

\begin{abstract}
The effect of Zn:Se ratio on the photoconducting properties of ZnSe thin films has been studied. The ZnSe thin films have been deposited onto glass substrates by the spray pyrolysis method, the substrate temperature kept at $430^{\circ} \mathrm{C}$ using mixed aqueous solutions of $\mathrm{ZnCl}_{2}$ and $\mathrm{SeO}_{2}$ with different $\mathrm{Zn}$ :Se ratios. Their electrical, structural, and photoconductivity properties have been studied. The values of optical bandgap have been determined from the absorption spectra.
\end{abstract}

PACS numbers: 78.66.Hf, 73.50.Pz

\section{Introduction}

Binary semiconductors are considered as important technological materials because of their potential applications in optoelectronic devices, solar cells, IR detectors and lasers $[1,2]$. Binary compounds of group IIB and group VIA elements, commonly referred to as II-VI compounds, have technologically important applications. Among these compounds, only cadmium telluride (CdTe) and zinc selenide (ZnSe) can be prepared in both $n$ - and $p$-type forms [3]. Thin films of these compounds are usually prepared by vacuum evaporation, chemical vapor deposition, sputtering and spray pyrolysis method $[4,5]$. Spray pyrolysis is widely used for the large-scale production of films owing to its low production cost and simplicity of operation.

Many efforts are currently directed to a new generation of photodiodes based on wide bandgap compound semiconductors. ZnSe meets this requirement because it has a wide bandgap (direct bandgap, $E_{\mathrm{g}}=2.7 \mathrm{eV}$ ) and is capable of emitting light in the blue-green region. ZnSe thin films have been usually grown by molecu-

*corresponding author; e-mail: bedir@gantep.edu.tr 
lar beam epitaxy $[6]$ and chemical vapor deposition $[7,8]$. On the other hand, several reports on the electrodeposition from aqueous solutions $[9,11]$ as well as from molten salts [12] have been published. However, little study has been reported about the sprayed ZnSe thin films. The high-quality, stoichiometry-controlled $p$-type ZnSe bulk crystals as well as $p$-type epitaxial layers have been produced by the temperature difference method under controlled vapor pressure (TDMCVP) [13]. And also the growth of sufficiently low resistivity $n$-type epitaxial layers on ZnSe substrates is applicable to realize high performance ZnSe $p-n$ junction directly formed on the high quality $p$-type ZnSe substrates. Although ZnSe is normally $n$-type, the as-grown electrodeposited films present $p$-type conductivity as inferred from the photoelectrochemical response [14]. Another interesting feature is that depending on the electrodeposition conditions, the films present either a gray or red coloration depending on whether they have an excess of $\mathrm{Zn}$ or Se, respectively.

In this paper, Zn:Se volume ratios were 0.2:1, 0.4:1, 0.6:1, 0.8:1, 1:1 for depositing ZnSe thin films by spray pyrolysis method at $430^{\circ} \mathrm{C}$ substrate temperature on the high cleaned glass substrates in the normal atmospheric conditions. The effect of Zn:Se ratio on photoconducting ZnSe films was studied by optical absorption and transmission, $I-V$ characteristics in dark and under light. It was observed that the $\mathrm{Zn}$ :Se ratio 0.2:1 gives the best results.

\section{Experimental details}

Spray pyrolysis involves the application of a fine mist of very small droplets containing the reactants onto the hot substrates in the atmospheric conditions. The critical operations of the spray pyrolysis technique are the preparation of uniform and fine droplets and the controlled thermal decomposition of these droplets.

The $\mathrm{ZnSe}$ thin films were prepared by spraying an aqueous solution of $\mathrm{ZnCl}_{2}$ and $\mathrm{SeO}_{2}$ on the high cleaned glass substrate kept at $430^{\circ} \mathrm{C}$. The atomization of the chemical solution into a spray of fine droplets is effected by the spray nozzle, with the help of compressed air by the air pump as carrier gas. The solution was sprayed through a nozzle onto glass substrates $\left(10 \times 10 \mathrm{~mm}^{2}\right)$ using air as carrier gas with a pressure of 1.5 bars. Totally, $100 \mathrm{~cm}^{3}$ of solution was used and sprayed for $45 \mathrm{~min}$. By varying the volume of $\mathrm{ZnCl}_{2}$ solution, $\mathrm{Zn}$ :Se ratio was varied as 1:1, $0.8: 1,0.6: 1,0.4: 1,0.2: 1$ and thin films were prepared in the normal atmospheric conditions. Details of the sample preparation are given elsewhere [15]. During the spraying process the substrates were heated by an electrical heater. The flow rate of the solution was kept at $5 \mathrm{~cm}^{3} \mathrm{~min}^{-1}$ and controlled by a flowmeter. The distance between the nozzle and the substrate was maintained at $35 \mathrm{~cm}$ and the substrate temperature of $430^{\circ} \mathrm{C}$ was controlled within $\pm 5^{\circ} \mathrm{C}$ by using an ironconstantan thermocouple. The annealing process was carried out by heating at $400^{\circ} \mathrm{C}$ during $60 \mathrm{~min}$ in an open air furnace. Electrical contacts were made through indium electrodes of thickness $750 \mathrm{~nm}$ on the surface of the thin films using vacuum 
evaporation technique. The thickness of the thin films was measured between 0.5 and $1.2 \mu \mathrm{m}$ using a Dektak 3030 profilometer.

Optical properties were studied by transmittance and optical absorption at room temperature, in the spectral range between 300 and $800 \mathrm{~nm}$, by using a UV-VIS double beam spectrophotometer. The crystallinity of the films was characterized by X-ray diffraction (XRD) using a Philips X-ray powder diffractometer provided with a $\mathrm{Cu}$ tube with $K_{\alpha}$ radiation at $\lambda=0.154 \mathrm{~nm}$ at an interval $32-40^{\circ}$. Dark and white light conductivity of the samples were measured in air at room temperature. The samples of the size $1.0 \mathrm{~cm} \times 1.0 \mathrm{~cm}$ were used. A dc voltage was applied up to $40 \mathrm{~V}$ and $500 \mathrm{~W}$ projection was used as a white light source.

\section{Results and discussion}

\subsection{Optical absorption and transmission studies}

The experiments on variation of bandgap as a function of $\mathrm{Zn}$ :Se ratio were carried out. From the absorbance data, the absorption coefficient $(\alpha)$ as a function of the photon energy was calculated and was plotted for the direct allowed transition by using the following formula:

$$
(\alpha h \nu)^{2} \propto h \nu-E_{\mathrm{g}}
$$

where $E_{\mathrm{g}}$ is the transition energy gap and $h \nu$ is the photon energy. Figure 1 shows the $(\alpha h \nu)^{2}$ versus $h \nu$ plots of ZnSe thin film with the ratio 0.2:1 as deposited $430^{\circ} \mathrm{C}$ substrate temperature and unannealed thin film.

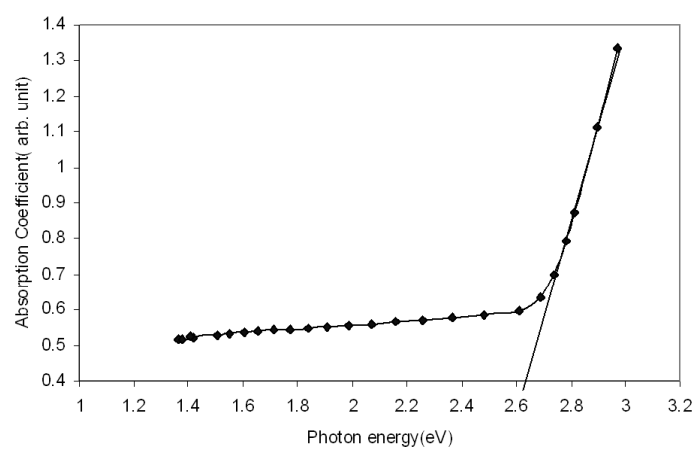

Fig. 1. The $(\alpha h \nu)^{2}$ versus $h \nu$ plot of unannealed ZnSe thin film with the 0.2:1 ratio.

It is clearly seen from Fig. 1 that there is some tailing in the bandgap below the absorption edge. This indicates that there is a high concentration of impurity states in the thin films which can cause a perturbation in the band structure with the result that the parabolic distribution of the states will be disturbed by a prolonged tail into the energy gap [16]. 
The tails in the optical spectra of the thin films could be a result of the broadening of the impurity levels due to their spatial overlap into a band. At high concentrations, the impurity band merges with the nearest intrinsic band. Therefore, the Fermi level will lie inside the parabolic portion of the appropriate band. Thus, less activation energy will be needed for the electrons to move from the Fermi level into the conduction band. The tails could also be due to ionized donors which could exert an attractive force on the valence band. The presence of impurity levels could affect the activation energy. The activation energy obtained for the thin films is given in Table I.

TABLE I

Estimated bandgap ' $E_{\mathrm{g}}$ ' and activation energy for differnt Zn:Se ratios in ZnSe thin films.

\begin{tabular}{c|c|c|c|c}
\hline \hline $\begin{array}{c}\text { Sample } \\
\text { number }\end{array}$ & $\begin{array}{c}\text { Zn:Se } \\
\text { ratio }\end{array}$ & $\begin{array}{c}\text { Unannealed thin } \\
\text { films, ' } E_{\mathrm{g}} \text { ' }[\mathrm{eV}]\end{array}$ & $\begin{array}{c}\text { Annealed thin } \\
\text { films, ' } E_{\mathrm{g}} \text { ' }[\mathrm{eV}]\end{array}$ & $\begin{array}{c}\text { Activation energy } \\
\text { with unannealed } \\
\text { thin films, } E_{\mathrm{a}}[\mathrm{eV}]\end{array}$ \\
\hline 1 & $0.2: 1$ & 2.65 & 2.63 & 0.59 \\
2 & $0.4: 1$ & 2.67 & 2.65 & 0.61 \\
3 & $0.6: 1$ & 2.69 & 2.67 & 0.63 \\
4 & $0.8: 1$ & 2.70 & 2.69 & 0.64 \\
5 & $1: 1$ & 2.71 & 2.70 & 0.65
\end{tabular}

The activation energy values obtained from the resistance measurements, therefore, give an indication of doped levels (trapped levels or additional energy levels) due to the presence of impurity atoms in the forbidden gap of the semiconducting thin films. Impurities and imperfections drastically affect the electrical properties of a semiconductor.

Figure 2A shows the transmission spectra of the ZnSe thin films as deposited at $430^{\circ} \mathrm{C}$ substrate temperature of unannealed thin films with different $\mathrm{Zn}$ :Se ratios. These films were applied to the annealing process by heating at $400^{\circ} \mathrm{C}$ during $60 \mathrm{~min}$ in an open air furnace. The transmission spectra for annealed thin films with different Zn:Se ratios are shown in Fig. 2B. And also after annealing, the direct bandgap of the ZnSe thin films was calculated. Thus the direct bandgap values for annealed and unannealed thin films with different $\mathrm{Zn}$ :Se ratios are listed in Table I. From transmission study of annealed and unannealed thin films, it was observed that ' $E_{\mathrm{g}}$ ' reduces with decreases in Zn:Se ratio. The ' $E_{\mathrm{g}}$ ' of Zn:Se at ratio $0.2: 1$ was found to be minimum. During annealing a selenium from the top surface gets evaporated resulting in selenium vacancies. This leads to diffusion of loosely bound selenium atoms along the grain boundary towards the surface. This is reflected into an increase in blue-green response [17]. 

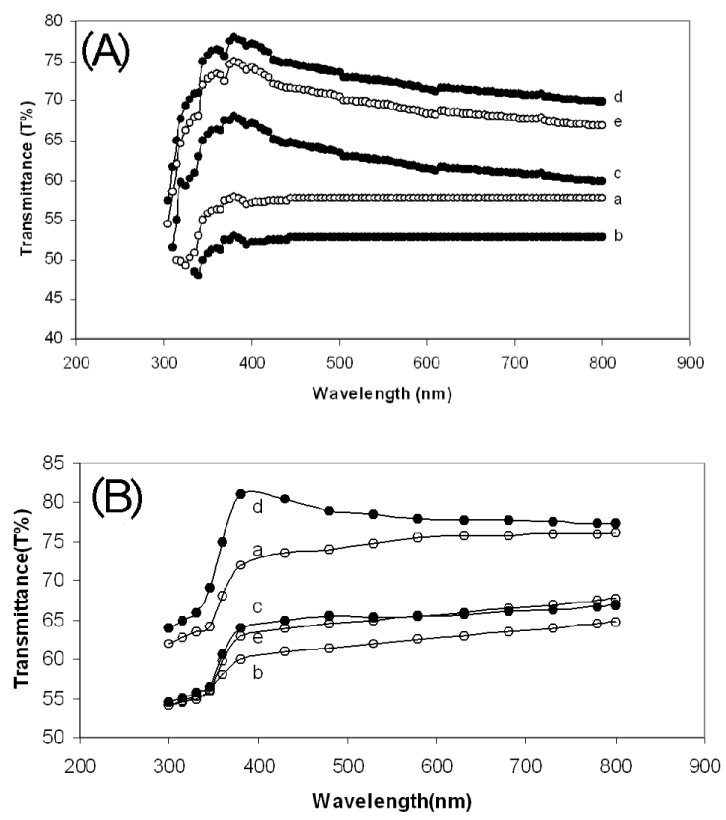

Fig. 2. Variation of optical transmittance $(T \%)$ with wavelength $(\lambda)$ for different Zn:Se ratios: (a) $0.2: 1$, (b) 0.4:1, (c) 0.6:1, (d) 0.8:1, and (e) 1:1 of unannealed (A)/annealed (B) thin films deposited at substrate temperature $430^{\circ} \mathrm{C}$.

\subsection{X-ray studies}

In order to study the crystalline nature of the thin films with different Zn:Se ratios, the XRD patterns were studied. The thin films were slow scanned between 32 to $40^{\circ}$. Figure $3 \mathrm{~A}$ and $\mathrm{B}$ shows such scans for the unannealed and annealed thin films with different $\mathrm{Zn}$ :Se ratios. It is observed that the XRD patterns of all ZnSe thin films show a most preferred orientation along (200) plane. Figure 3B shows the $\mathrm{XRD}$ of the ZnSe thin films after annealing at $400^{\circ} \mathrm{C}$ for $60 \mathrm{~min}$. After annealing, at $430^{\circ} \mathrm{C}$ substrate temperature with different $\mathrm{Zn}$ :Se ratios the crystallinity of the as-deposited thin films improved which is observed from the occurrence of sharp, intense peaks with a reduced full width at half maximum. This confirms that upon annealing, the crystallinity of the film is increased due to recrystallization of the as-deposited unannealed thin films. It has been observed that the crystallinity is increased upon annealing for the film deposited with different Zn:Se ratios.

It is clearly seen that the thin films are nanocrystalline. The grain size for (200) diffraction peak (shown in Fig. 3A) for different Zn:Se ratio thin films was calculated using the following relation:

$$
d=\frac{\lambda}{D} \cos \theta
$$

where $d$ is the crystal size, $\lambda$ - the X-ray wavelength used, $D$ - the angular line width of half maximum intensity, $2 \theta-$ the Bragg's angle [18]. The calculated 

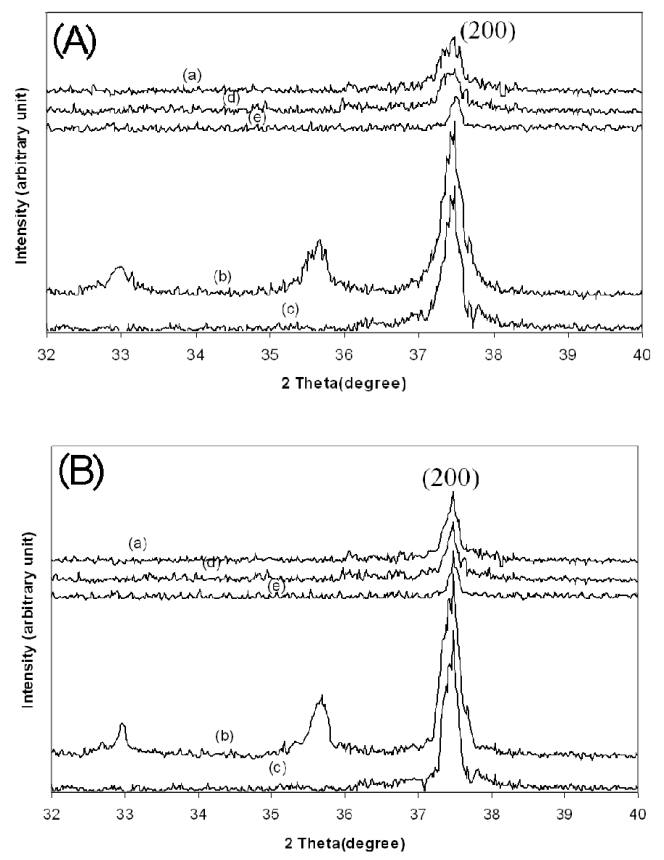

Fig. 3. X-ray diffraction patterns for different unannealed (A)/annealed (B) films Zn:Se ratios: (a) $1: 1$, (b) $0.2: 1$, (c) $0.4: 1$, (d) $0.6: 1$, and (e) 0.8:1.

grain sizes of the annealed and unannealed thin films for different Zn:Se ratios are given in Table II. From Table II, it was observed for unannealed films that as $\mathrm{Zn}: \mathrm{Se}$ ratio decreases, the grain size increases. And also the grain size of the ZnSe thin films after annealing at $400^{\circ} \mathrm{C}$ for 60 min increases slightly by the annealing treatment [19]. Thus it was observed that this increase in grain size causes an increase in the blue-green response by a small amount, due to a decrease in bandgap ' $E_{\mathrm{g}}$ '.

TABLE II

Grain sizes for different $\mathrm{Zn}$ :Se ratios of unannealed and annealed ZnSe thin films.

\begin{tabular}{c|c|c|c}
\hline \hline $\begin{array}{c}\text { Sample } \\
\text { number }\end{array}$ & Zn:Se ratio & $\begin{array}{c}\text { Grain size }(\AA) \\
\text { for unannealed films }\end{array}$ & $\begin{array}{c}\text { Grain size }(\AA) \\
\text { for annealed films }\end{array}$ \\
\hline 1 & $0.2: 1$ & 32 & 33.4 \\
2 & $0.4: 1$ & 29 & 30.8 \\
3 & $0.6: 1$ & 23 & 24.9 \\
4 & $0.8: 1$ & 23 & 24.6 \\
5 & $1: 1$ & 22 & 23.2
\end{tabular}




\subsection{Photoconductivity study}

\subsubsection{Dark and photoconductivity studies}

Different Zn:Se ratio unannealed thin films were formed and their dark conductivity and photoconductivity were measured at room temperature under identical conditions. The $I-V$ curves of dark conductivity and photoconductivity for Zn:Se ratio as 0.2:1 is shown in Fig. 4. The dark and photoconductivity for different Zn:Se ratios measured at $40 \mathrm{~V}$ dc are given in Table III.

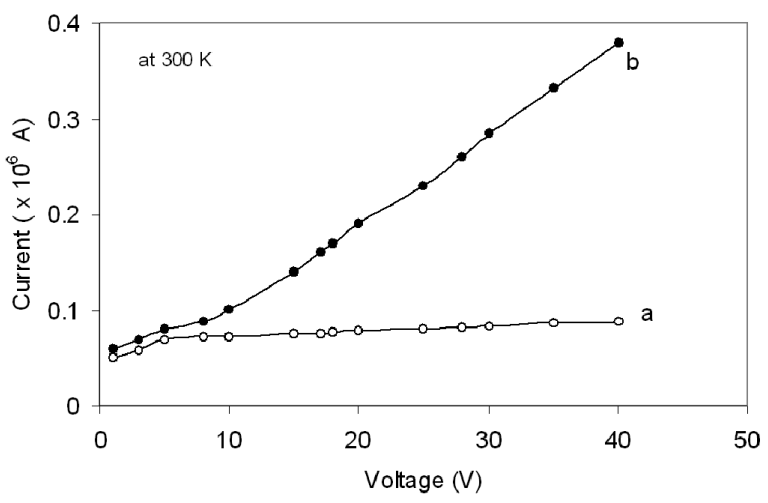

Fig. 4. Variation of current $(I)$ with voltage for Zn:Se ratio as 0.2:1; (a) dark conductivity, (b) photoconductivity at $300 \mathrm{~K}$.

TABLE III

Dark conductivity and photoconductivity for different Zn:Se ratios.

\begin{tabular}{c|c|c|c|c|c|c}
\hline \hline \multirow{2}{*}{$\begin{array}{c}\text { Serial } \\
\text { number }\end{array}$} & Measured parameters & \multicolumn{5}{|c}{ Zn:Se ratio } \\
\cline { 3 - 7 } & {$\left[10^{-6} \Omega \mathrm{cm}^{-1}\right]$} & $0.2: 1$ & $0.4: 1$ & $0.6: 1$ & $0.8: 1$ & $1: 1$ \\
\hline \hline 1 & Dark conductivity $\sigma_{\mathrm{d}}$ & 1.15 & 1.11 & 1.05 & 1.0 & 0.96 \\
\hline 2 & Photoconductivity $\sigma_{\mathrm{L}}$ & 2.25 & 2.05 & 1.86 & 1.70 & 1.55
\end{tabular}

It was observed that as the Zn:Se ratio decreases, the photoconductivity increases. This is because of ' $E_{\mathrm{g}}$ ' reduction and an increase of sample absorption. When such films are excited in light, a large number of electrons are found to be available in conduction band for the same intensity of light. The sample having $\mathrm{Zn}$ :Se ratio as $0.2: 1$ is to be more photoconductive.

\subsubsection{Photoconductivity rise and decay}

The rise and decay of the photoconductivity for different $\mathrm{Zn}$ :Se ratio unannealed thin films were studied in air for the same light intensity with an applied field $40 \mathrm{~V}$ dc. Figure 5 shows three continuous successive cycles with rise times 
of $20 \mathrm{~min}$ and decay until the original dark current was reached for $\mathrm{Zn}$ :Se ratio as $0.2: 1$. In the first cycle of excitation the photocurrent increases slowly but not linearly with time. The linear nature of the photocurrent with time for the first cycle of excitation was observed in ZnSe films as reported by Nair et al. [20]. No steady state photocurrent was obtained even after $20 \mathrm{~min}$. However, in the second and third cycles the rise is much steeper and the curve bends towards steady state value. It was observed that both photocurrents have been increased, the former being very large.

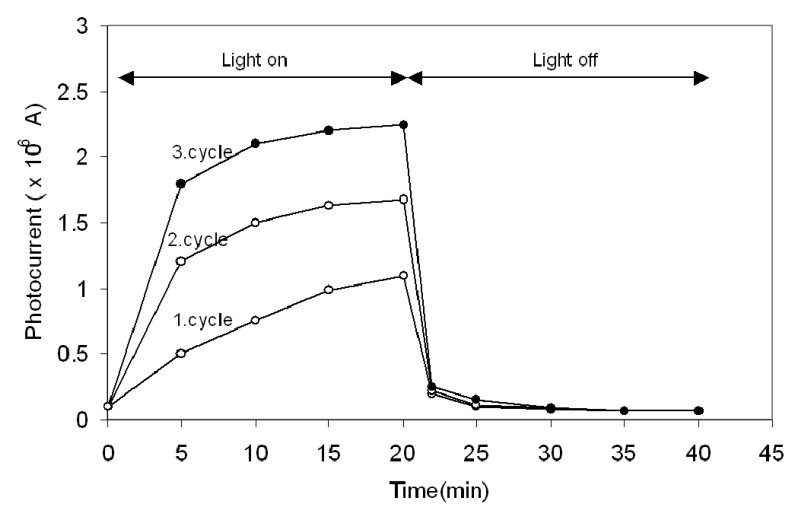

Fig. 5. The rise and decay of the photocurrent with time for three successive cycles for $\mathrm{Zn}:$ Se ratio as 0.2:1.

It is well known that oxygen impurity is found as a background impurity in many material because of its easily diffusing properties into the crystal lattice during the production of samples or during the annealing stage of the samples in air at elevated temperatures. The oxygen ions play a critical role in the performance of electroluminescent devices and, therefore, the role of oxygen impurities in these materials has been studied over the last 40 years. The other residual impurity is the $\mathrm{Cl}$ ions that come from the used salts in the spraying solutions. Oxygen impurities are known to be first physically adsorbed at the grain boundaries and on the ZnSe surface. In the present investigation, as the films were prepared by the spray pyrolysis method, oxygen molecules are assumed to be physically adsorbed at the grain boundaries and on the ZnSe surface. They then become chemisorbed having captured a conduction electron, which binds them to the surface. The energy levels of such bound electron are sufficiently below the conduction band that in the dark, the rate of escape of electrons from the surface to the conduction band is negligible. Holes move to the surface under illumination and are captured by negatively charged oxygen molecules, giving neutral molecules which are weakly bound and can escape from surface. This process is responsible for the low values of both the dark current and photocurrent in the first cycle of excitation. In the second and successive cycles a larger number of oxygen molecules are desorbed, resulting 
in the faster rise time and higher photocurrent [21]. In short, the chemisorbed oxygen at the intergrain region builds up repulsive potential for the transport of electrons, leading to very low electron drift mobilities and hence produces only very small electric currents. Illumination produces electron-hole pairs, of which the holes neutralize the repulsive potential at the grain boundaries and leads to an exponential increase in the electron mobility. This results in a high photocurrent under illumination which combined with the very low current in the dark produces a high photosensitivity in the thin films.

\section{Conclusion}

The effects of Zn:Se ratios on the absorption and photoconducting properties of sprayed ZnSe thin films are analyzed. The values of optical bandgap were determined from the absorption spectra. It was observed that the decrease in bandgap energy with the increased Se content in the thin film can be attributed to the increase in the grain size of the thin film. The low bandgap energy obtained for the $0.2: 1$ ratio of $\mathrm{Zn}$ :Se could be attributed to the small grain size in other different thin films. The small grain size leads to electrical isolation of the individual grains, or quantum well structure. It is found that the ZnSe thin films show high photoconductivity properties and this photoconductivity is enhanced for the thin film samples prepared at 0.2:1 ratio of $\mathrm{Zn}$ :Se.

\section{References}

[1] G. Riveros, H. Gomez, R. Henriguez, R. Schrebler, R.E. Maratti, E.A. Dalchiele, Sol. Energy Mater. Sol. Cells 70, 255 (2001).

[2] V. Kumar, T.P. Sharma, Opt. Mater. 10, 253 (1998).

[3] L. Ting Chu, S.C. Shirley, Solid-State Electron. 38, 533 (1995).

[4] L.M. Caicedo, G. Cediel, A. Dussan, J.W. Sandino, C. Calderon, G. Gordillo, Phys. Status Solidi B 220, 249 (2000).

[5] B. Su, K.L. Choy, Thin Solid Films 102, 361 (2000).

[6] H. Goto, T. Ido, A. Takatsuka, J. Cryst. Growth 529, 214 (2000).

[7] S. Fujita, Y. Matsuda, A. Sasaki, J. Cryst. Growth 231, 140 (1984).

[8] C.D. Lukhande, P.S. Patil, A. Ennaoui, H. Tributsch, Appl. Surf. Sci. 294, 123 (1998).

[9] J.P. Rai, Sol. Energy Mater. 30, 376 (1993).

[10] A. Lizzo, M.A. Tagliente, L. Caneve, S. Scaglione, Thin Solid Films 8, 368 (2000).

[11] K. Singh, R.K. Pathak, Electrochem. Acta 39, 2683 (1994).

[12] S. Sanchez, C. Lucas, G.S. Picard, M.R. Bermejo, Y. Castrillejo, Thin Solid Films 107, 361 (2000).

[13] F. Sakurai, M. Motozawa, K. Suto, J. Nishizawa, J. Cryst. Growth 75, 172 (1997). 
[14] A.P. Samantilleke, M.H. Boyle, J. Young, I.M. Dharmadasa, J. Mater. Sci. 9, 289 (1998).

[15] M. Öztaş, M. Bedir, P.J. Appl. Sci. 1(2), 214 (2001).

[16] R.K. Nkum, A.A. Adimado, H. Totoe, Mater. Sci. Eng. B 55, 102 (1998).

[17] C.D. Lokhande, S.H. Pawar, Solid State Commun. 44, 1137 (1982).

[18] S.S. Kale, C.D. Lokhande, Mater. Chem. Phys. 62, 103 (2000).

[19] M.J. Mangalam, K.N. Rao, N. Rangarajan, C. Suryanarayana, Ind. J. Pure Appl. Phys. 7, 628 (1969).

[20] P.K. Nair, M.T.S. Nair, V.M. Gurcia, O.L. Arenas, Y. Pena, A. Castillo, I.T. Ayala, O. Gomazdaza, A. Sanchez, J. Campus, H. Hu, R. Suarez, M.E. Rincon, Sol. Energy Mater. Sol. Cells 52, 313 (1998).

[21] M. Öztaş, Ph.D. Thesis, Gaziantep University, Gaziantep (Turkey) 1996, p. 175. 